\title{
Effect of Pore Size, Lubricant Viscosity, and Distribution on the 2 Slippery Properties of Infused Cement Surfaces
}

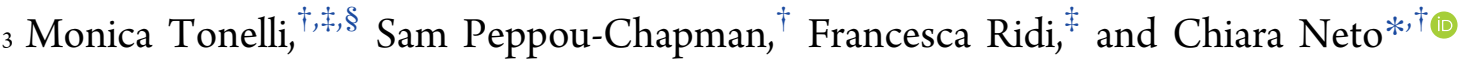 \\ $4{ }^{\dagger}$ School of Chemistry and University of Sydney Nano Institute, The University of Sydney, Sydney, New South Wales 2006, Australia \\ 5 "Department of Chemistry “Ugo Schiff” \& CSGI, University of Florence, Sesto Fiorentino 50019, Florence, Italy
}

6 S Supporting Information

\begin{abstract}
The fabrication of slippery lubricant-infused porous surfaces (SLIPS) usually requires the use of structured substrates, with specifically designed micro- and nanoroughness and complementary surface chemistry, ideally suited to trap lubricants. It is not yet established whether a random roughness, with a range of pores with a variable size reaching deep into the bulk of the material, is suitable for successful infusion. In this study, a highly porous material with random and complex roughness, obtained by using portland cement (the most common type of cementitious material), was tested for its potential to act as a SLIP surface. Atomic force microscopy meniscus measurements were used to investigate

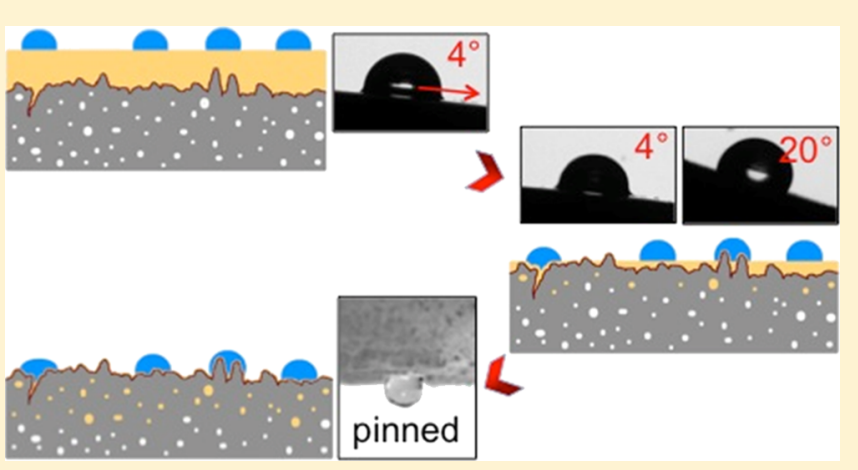
the distribution of lubricants on the surface upon subsequent stages of depletion because of the capillary absorption of the lubricant within the porous structure. Factors such as curing time of the cement paste, time since infusion, and lubricant viscosity were varied to identify the conditions under which infusion could be considered successful. A sensitive method to evaluate the penetration of liquid (low-temperature differential scanning calorimetry) was used, which could be applicable to many porous materials. The optimized infusion of cement surfaces ultimately resulted in the desired hallmarks of SLIPS, that is, high water repellence and slipperiness, effective for several weeks, reduced water permeability, and icephobicity.
\end{abstract}

27 Slippery liquid infused porous surfaces (SLIPS) are materials 28 inspired by the slippery properties of the trumpet pitcher plant, 29 which uses controlled surface microroughness to trap thin 30 water films and make its pitcher slippery to insects. SLIPS have 31 been shown to have self-cleaning, ${ }^{1,2}$ anti-adhesive, ${ }^{3}$ anti32 icing, ${ }^{4-10}$ anticorrosion, ${ }^{11}$ antibiofouling, ${ }^{12-15}$ and potential 33 drag-reducing properties. ${ }^{11-14}$ SLIPS are usually made of 34 structured hydrophobic materials, where the structure is 35 carefully controlled and optimized on the nano- and 36 microscale, in order to obtain successful and durable 37 infusion. $^{13,16,17}$ The lubricant infusion is guided by capillary 38 wicking within the employed surface features, and therefore 39 benefits from control over both size and surface chemistry. ${ }^{18}$ 40 For example, there is some evidence that nanoscale features are 41 more effective than microscale features in retaining lubricants 42 upon spinning at high rates. ${ }^{19}$

43 Much of the research on SLIPS has focused either on 44 fabrication methods or on the engineering aspects for specific 45 applications. Only more recently, there have been publications 46 which explore fundamental aspects of these materials, for 47 example, the stability of lubricant films on surfaces with and 48 without the structure, in air, and in the liquid to be 49 repelled. ${ }^{15,20-24}$ There still remains much work to be done 50 to fully understand the mechanisms that underpin the function of SLIPS. It is unclear, for example, exactly how the lubricant is 51 displaced over time between and on top of micro- and 52 nanostructures because of different depletion mechanisms that 53 are likely to occur. This lack of understanding means that it is 54 also unclear what range of surface structure is suitable for 55 infusion, and whether real-world surfaces are likely to succeed 56 in obtaining slippery properties.

The preparation of effective slippery liquid-infused cement 58 surfaces has not yet been investigated, although the research on 59 protective treatments of cement-based structures is exten- 60 sive. $^{25-28}$ Nowadays, self-cleaning treatments on cement-based 61 surfaces are widely used but they rely on the obtainment of 62 hydrophobic/superhydrophobic coatings, whereas the idea 63 that cement surfaces can be infused with silicone oils and 64 become SLIPS, thermodynamically more stable and potentially 65 more robust liquid-repellent surfaces, ${ }^{12}$ is proposed here for 66 the first time. SLIPS are able to prevent the adhesion of water, 67 organic solvents, and solutions containing contaminants, 68 including bacteria and larger marine organisms. ${ }^{13}$ SLIPS are 69 thermodynamically more stable and potentially more robust 70 liquid-repellent surfaces than superhydrophobic ones, ${ }^{2,18}$ but it 71

Received: November 19, 2018

Revised: January 16, 2019 
72 is not obvious that the lubricant infusion could be efficiently 73 performed on a random roughness with a high number of 74 nanoscale and microscale pores, such as that intrinsically 75 present in cement.

76 Here, for the first time, the porous and random structure of 77 hardened cement paste is shown to be suited to achieve 78 effective infusion, leading to slippery surfaces. Despite being 79 self-assembled and somewhat uncontrolled, the characteristic 80 micro- and nanostructure of cement surfaces does retain the 81 lubricant as long as the curing time and infusion process are 82 optimized for this purpose. This treatment results in high water 83 repellence and slipperiness (as determined by low sliding 84 angles), effective over several weeks, reduced water pene85 tration, and reduction of freezing temperature of water. In this 86 paper, a differential scanning calorimetry (DSC) method was 87 established that allowed us to study how the degree of infusion 88 affects the degree of penetration of water in the pores and its 89 freezing. Moreover, calorimetry was used, for the first time, to 90 quantitatively evaluate the icephobic properties of SLIPS91 treated surfaces by looking at the temperature of ice 92 nucleation. Infused cement surfaces not only absorb less 93 water but they also self-clean, thus providing protection and 94 reduced restoration costs. The conditions that underpin the 95 stability of the infusing lubricant layer on the surface were 96 investigated by the optimization of the infusion parameters. By 97 performing atomic force microscopy (AFM) meniscus force 98 measurements, the lubricant film thickness was mapped locally 99 and over time, which was crucial in establishing the point of 100 failure of the surfaces.

\section{MATERIALS AND METHODS}

102 Cement Paste Preparation and Characterization. A 103 commercial cement made by Cement Australia was used: 104 general purpose cement type GP. This cement (up to $50 \%$ of 105 the fresh dry material $<10 \mu \mathrm{m}$ ) has the following composition: $106<97 \%$ Portland clinker, $2-5 \%$ gypsum, $0-7.5 \%$ limestone, and $1070-1 \%$ calcium oxide. The cement pastes here investigated were 108 prepared with water/cement ratio $=0.4$. After mixing $\sim 20 \mathrm{~g}$ of 109 cement with water for $3 \mathrm{~min}$ by hand, the pastes were 110 transferred into polypropylene molds $(0.5 \mathrm{~cm}$ diameter $\times 0.3$ $111 \mathrm{~cm}$ height) and allowed to cure at room temperature. The use 112 of molds allowed the preparation of flat reproducible samples, 113 without affecting the physical-chemical properties of the 114 surfaces, which are strictly related to the microstructure. Static 115 contact angle and roll-off angle of water were measured (KSV 116 Cam 200) at various stages before and after silanization and/or 117 infusion, using $10 \mu \mathrm{L}$ droplets. The reported water static 118 contact angle and sliding angle values are the average of at least 119 six droplets on different regions of two samples. The hydration 120 kinetic was monitored (DSC Q2000 calorimeter, TA Instru121 ments) following a method already reported in the 122 literature, ${ }^{23,24,29}$ and is provided in the Supporting Informa123 tion. Surface area and pore size distribution were characterized 124 by nitrogen gas adsorption [Coulter SA 3100, Brunauer125 Emmett-Teller (BET) and Barrett-Joyner-Halenda (BJH) 126 methods]. Pore volume percentage distribution was calculated 127 from the adsorption curve.

128 Surface Silanization. A container was flushed with 129 nitrogen gas to remove oxygen and humidity and the cement 130 samples were placed on a Teflon base inside the container. 131 Droplets of a volatile silane $(\sim 100 \mu \mathrm{L}$ of dichlorodimethylsi132 lane, DCDMS, Sigma-Aldrich) were injected into the bottom 133 of the container without touching the samples. The sealed container was kept at room temperature for about $3 \mathrm{~h}$, allowing 134 silane vapor to react with the surface of the cement pastes. 135 Then, the samples were washed with acetone to remove 136 unbound silane. $^{30}$

Surface Infusion. Infusion of silicone oil into samples was 138 performed manually by spreading silicone oil over the surface. 139 Samples were kept horizontal at room temperature. Table 1140 tl summarizes the information on the silicone oils used (Aldrich). 141

Table 1. Different Silicone Oils Used for Infusion into the Cement Surfaces $^{a}$

\begin{tabular}{ccc}
$\begin{array}{c}\text { viscosity of the silicone oil } \\
(\mathrm{cSt})\end{array}$ & $\begin{array}{c}\text { molecular weight } \\
(\mathrm{g})\end{array}$ & $\begin{array}{c}\text { volume used for the infusion } \\
(\mu \mathrm{L})\end{array}$ \\
\hline 10 & $\sim 1250$ & 100 \\
350 & $\sim 14000$ & 100 \\
$1 \mathrm{k}$ & $\sim 30000$ & 100 \\
$10 \mathrm{k}$ & $\sim 63000$ & 40 \\
$20 \mathrm{k}$ & $\sim 73000$ & 40
\end{tabular}

${ }^{a}$ Weight average molecular weight was estimated using the MarkHouwink correlation viscosity/molecular weight. $^{31}$

Coating Thickness. The thickness of the 10k cSt silicone 142 oil coating was measured at different stages via AFM meniscus 143 force measurements (Asylum MFP-3D AFM, force mapping 144 mode) using Multi75AL-G cantilevers (budget sensors, 145 resonant frequency $75-80 \mathrm{kHz}, k=1-7 \mathrm{~N} / \mathrm{m}) .^{.6}$

Water Permeability in the Porous Structure. The 147 permeation of water was quantified by DSC (Q2000 TA 148 Instruments) by looking at the freezing behavior of water 149 confined in the porous structure. ${ }^{32-34}$ This method provides 150 information on the amount of water present in different parts 151 of the microstructure; the size of the pores in the micro- 152 structure can be distinguished based on the freezing point of 153 the water they contain. Three cement samples at 7 days of 154 hydration were investigated to quantify the water penetration 155 into the pores of the cement paste. One sample was SLIPS- 156 treated (silanised and infused with silicone oil 10k cSt) and the 157 others were left untreated. Small pieces (milligrams) of one of 158 the untreated sample and of the SLIPS-treated sample were 159 separately dipped in water. After 1 day, the exterior of the 160 samples was dried and the samples were placed in aluminum 161 pans. An untreated sample, used as a reference, was analyzed 162 without dipping it into water. The experiments were carried 163 out with the following temperature program: equilibrate to 5164 ${ }^{\circ} \mathrm{C}$; cooling ramp from 5 to $-80{ }^{\circ} \mathrm{C}$ at $0.5{ }^{\circ} \mathrm{C} / \mathrm{min}$; and 165 heating ramp from -80 to $+10{ }^{\circ} \mathrm{C}$ at $0.5{ }^{\circ} \mathrm{C} / \mathrm{min}$. The water 166 freezing peaks were integrated, and each area was used to 167 quantify the amount of water involved, by using as standard 168 enthalpy the estimation of $\Delta H_{0}$ by Hansen et al. ${ }^{35}$ at the 169 temperature of the maximum.

Ice Nucleation. The cement paste was transferred in two 171 DSC pans soon after mixing and cured at $20{ }^{\circ} \mathrm{C}$. Then, at 7172 days of hydration, one sample was silanised and infused and 173 the other was left untreated. In both cases, a $5 \mu \mathrm{L}$ droplet of 174 deionized water was added on the surface, and the pan was 175 sealed and repeatedly cooled and thawed. To evaluate the 176 icephobic properties of the SLIPS-treated surface in compar- 177 ison with the untreated one, and taking into account the 178 stochastic nature of nucleation of super cooled water, 150179 freeze-thawing cycles were carried out (DSC, Q2000 TA 180 Instruments). Following a protocol reported in the literature 181 for the study of the inhibition of ice nucleation, ${ }^{36,37}$ the 182 
183 thermal cycle was: ramp from 20 to $0{ }^{\circ} \mathrm{C}$ at $20^{\circ} \mathrm{C} / \mathrm{min}$; ramp 184 from 0 to $-40{ }^{\circ} \mathrm{C}$ at $5{ }^{\circ} \mathrm{C} / \mathrm{min}$; ramp from -40 to $+30{ }^{\circ} \mathrm{C}$ at $18520{ }^{\circ} \mathrm{C} / \mathrm{min}$; and equilibrate at $30{ }^{\circ} \mathrm{C}$. In each run, the 186 temperature at which the water froze was measured.

\section{RESULTS AND DISCUSSION}

188 Wettability. Cement samples were prepared and allowed to 189 cure for different times (1-40 days). Hardening of cement 190 pastes results from the reaction between the anhydrous 191 calcium silicates and aluminates that constitute the cement 192 powder and water, mostly happening in the early stage of 193 curing, which leads to the formation of an amorphous binder 194 phase with a porous structure. Figure 1 shows a schematic of

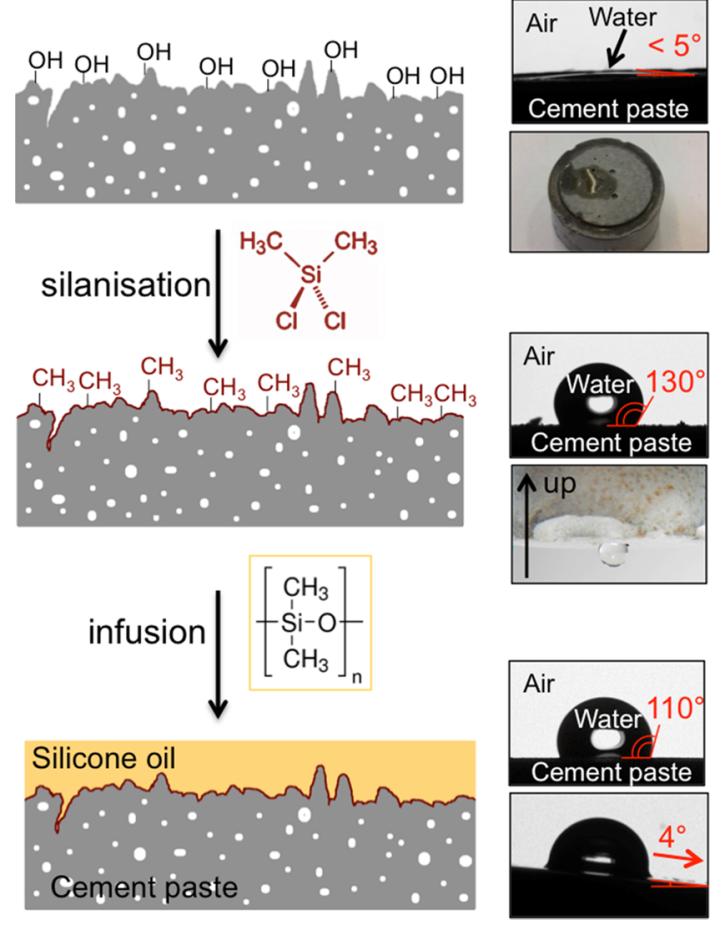

Figure 1. Schematic representation of the preparation of SLIPS based on cement with optical micrographs, showing static water contact angles and roll-off angles at each stage of surface modification.

195 the surface treatment that followed. The static water contact 196 angle on plain cement paste surfaces was found to be 197 consistently below $5^{\circ}$, with the water droplets becoming 198 absorbed entirely by the surface within a few seconds of 199 deposition. To increase the affinity of silicone oil for the 200 cement surface under water, ${ }^{13}$ the samples were hydro201 phobised with DCDMS. During silanization, hydroxyl groups 202 and hydrated species on the surface of the cement paste 203 covalently bond the silane agents, creating a new functionalized 204 surface with exposed $-\mathrm{CH}_{3}$ groups. ${ }^{30,38}$ After silanization, the 205 surface is hydrophobic (water contact angle around $130^{\circ}$ ), but 206 water droplets still remain pinned onto the surface (no sliding 207 off of droplets occurred, even upon surface inversion). 208 Following infusion with silicone oil, a hydrophobic surface is 209 obtained (water contact angle around $110^{\circ}$ ) which has slippery 210 properties, as established based on very low watersliding angles $211\left(\approx 4^{\circ}\right)$. The surfaces remained slippery to water droplets for 212 weeks after infusion, as described below.

213 Silicone oils of different viscosities were used for the infusion 214 of cement surfaces (at 7 days of curing), and the resulting water-sliding angle was measured as a function of time because 215 of infusion. Sliding angles were used as a qualitative measure of 216 surface slipperiness, as often done in the literature. ${ }^{3}$ As contact 217 line pinning is a nonequilibrium process, a statistical measure 218 of sliding angles is illustrated in Figure 2: a full symbol $219 \mathrm{f} 2$ corresponds to $>90 \%$ incidence of that sliding angle value 220 being measured over at least six measurements; an empty 221 symbol corresponds to $<10 \%$ incidence, and the semifilled 222 symbol corresponds to intermediate incidence. The values of 223 water static contact angle with time are reported in Figure S1 224 in the Supporting Information. The most viscous silicone oil 225 used $(20 \mathrm{k} \mathrm{cSt})$ produced good slippery properties, with low 226 water-sliding angle values $\left(<4^{\circ}\right)$; these surfaces were also the 227 most durable, with similar low sliding angle values lasting over 228 periods longer than 4 weeks. Silicone oil of $10 \mathrm{k} \mathrm{cSt}$ was entirely 229 effective in inducing low sliding angles at short times $(<2230$ weeks), but the sliding angles increased gradually with time. 231 When using lower viscosity silicone oils (10, 350, and 1000232 $\mathrm{cSt}$ ), water droplets pinned to the surfaces soon after infusion 233 in both silanised and nonsilanised cases (sliding contact angle 234 $>180^{\circ}$ ). With low viscosity silicone oils, the oil was quickly 235 absorbed by capillarity in the cement texture, causing the 236 failure of the slippery properties (i.e. water droplets pinned 237 onto the surface) after 1, 3, and 7 days when using 10, 350, and 238 $1000 \mathrm{cSt}$ silicone oil, respectively (Figure 2A). This behavior is 239 expected, as a liquid in contact with a porous material is 240 absorbed by capillary forces more quickly, the less viscous the 241 liquid is. ${ }^{28}$ When not silanised (Figure 2B), surfaces reached 242 the failure point faster, whereas the silanization treatment 243 produced a more favorable infusion and, consequently, a more 244 robust wettability modification with time (Figure 2A). This 245 effect is particularly evident when using 10k cSt silicone oil. 246 When not silanised, this sample showed a slow and gradual 247 transition from a sliding angle of about $4^{\circ}$ to a sliding angle 248 $>180^{\circ}$ at $10-17$ days, during which slippery and nonslippery 249 regions coexisted on the sample (Figure $2 \mathrm{~B}$ ). When silanised 250 and infused, the sample showed a gradual increase of the 251 sliding angle at about 2 weeks after infusion (Figure 2A, 15-16 252 days). As previously observed by the authors for infused 253 polymer surfaces of different chemistries, ${ }^{2,13}$ hydrophobization 254 of the cement is a required step for successful and robust 255 infusion. $^{39}$

Treatment Optimization. Cement-based materials have a 257 complex multiscale structure that changes with curing time. 258 The exact microstructure is influenced by the formation of the 259 principal reaction product, calcium silicate hydrate, $(\mathrm{CaO})_{x}-260$ $\left(\mathrm{SiO}_{2}\right)_{y}-\left(\mathrm{H}_{2} \mathrm{O}\right)$. The aging conditions have a significant effect 261 on the hydration of the cement paste and the porosity of the 262 paste, which in turn strongly influence its mechanical 263 properties, durability, permeability, and diffusivity. ${ }^{40,41}$ As 264 infusion treatments should be performed once the cement 265 paste set and the microstructure has almost completely formed, 266 monitoring the hydration kinetics is paramount.

Following the free water index, which is directly related to 268 the degree of hydration, as a function of the time (Figure S2) 269 allowed us to determine that the cement microstructure, 270 because of the growing hydrated phases, was completely 271 formed within less than 1 week $(\sim 120 \mathrm{~h})$. To further 272 investigate what the ideal cure time is, wettability and nitrogen 273 adsorption were investigated.

274

Figure 3 shows the sliding angle versus time since infusion $275 \mathrm{f} 3$ for a sample silanised and infused with $10 \mathrm{k}$ cSt silicone oil at 276 different times of curing (corresponding water static contact 277 


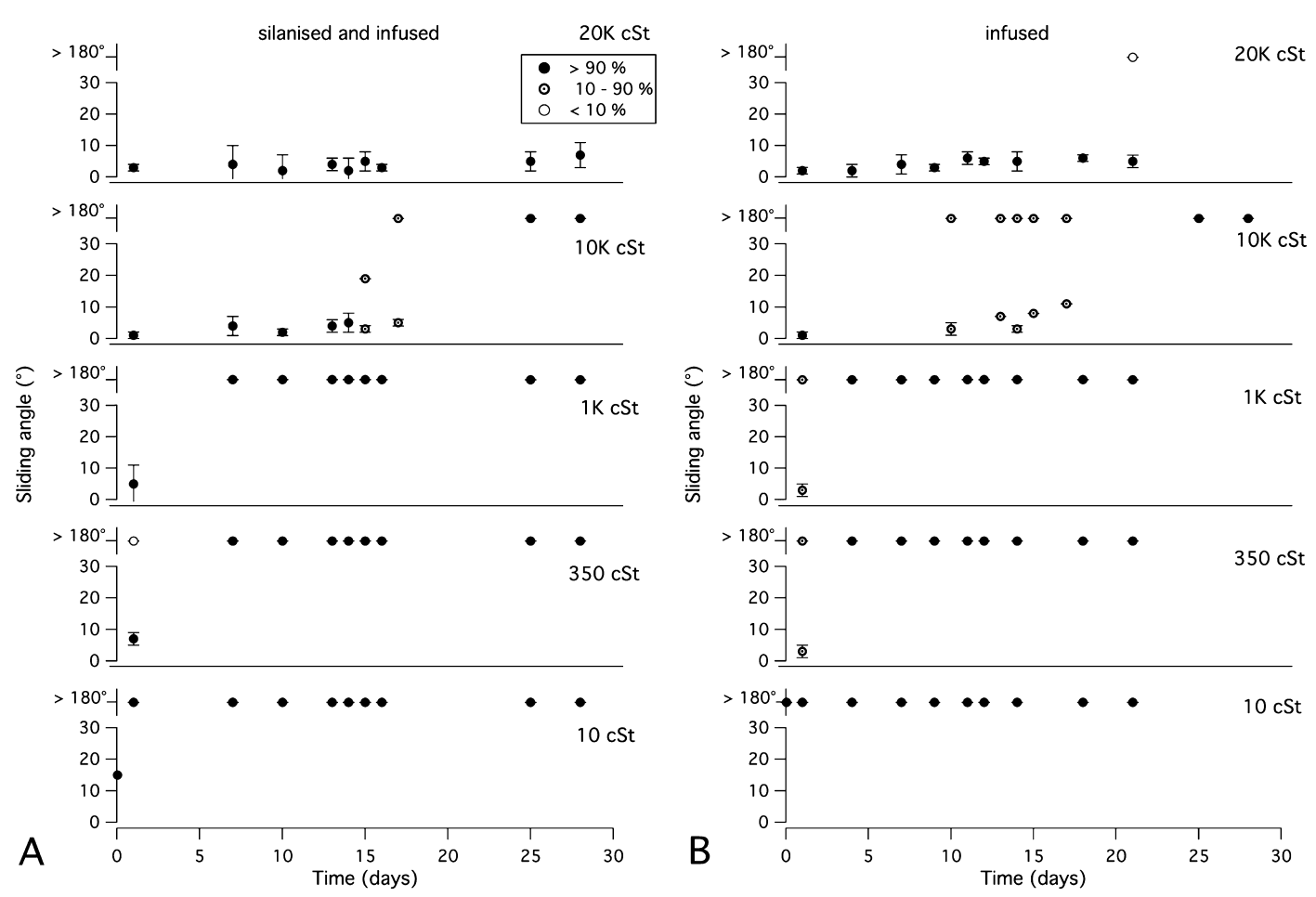

Figure 2. Sliding angle of a water droplet on cement surfaces over time since infusion, using silicone oils of different viscosities. (A) Cement surfaces after SLIPS treatment (silanised and infused); (B) surfaces infused without silanization. Full circles represent values measured with more than $90 \%$ incidence for that system; semifilled circles represent values measured at between 10 and $90 \%$ incidence; and empty circles represent less than $10 \%$ incidence for that system.

278 angle reported in Figure S3). When infusion occurred after 1 279 day of curing of the cement paste, the system lost its slippery 280 properties immediately, within the first day after infusion 281 because of the presence of large interconnected pores in the 282 paste, which accelerate the drainage of the silicon oil away 283 from the surface and into the bulk of the cement (see nitrogen 284 adsorption data later). When infused after 7 or 15 days of 285 curing, the surfaces maintained their slippery properties for 286 longer times, about 2 weeks after infusion, transitioning 287 gradually to a hydrophobic surface onto which water droplets 288 remained pinned.

289 Through porosimetry, the surface area and pore size 290 distribution were measured at different curing times. Figure 2914 shows the adsorption/desorption isotherms and the pore 292 volume percentage distribution for cement pastes aged 1, 7, 293 and 15 days, and Table 2 summarizes the BET surface area and 294 total pore volume calculated from the figure. All the 295 physisorption isotherms (Figure 4A) are compatible with 296 macroporous systems of type $\mathrm{II}^{42}$ and the hysteresis loops can 297 be classified as type H3, which is associated with capillary 298 condensation. ${ }^{43}$ The isotherms are compatible with the 299 existence of aggregates that give rise to polydisperse porosity 300 in the range from micropores to macropores (pore size $>50$ $301 \mathrm{~nm}) .{ }^{44}$ In cement pastes, the microstructure evolves through 302 the progressive closure of porosity, called depercolation 303 threshold, which in turn affects the transport properties that 304 in most degradation mechanisms govern the rate of damage. ${ }^{34}$ 305 The hysteresis loop at 1 day of curing is very narrow, whereas 306 after 7 and 15 days, the pastes show a wide loop, confirming 307 the increase of mesopore volume that mostly occurs in the first 308 week. The distribution of pore size dimensions (Figure 4B) 309 changed over time. After 1 day of curing, a wide distribution of 310 pore size between 55 and $165 \mathrm{~nm}$ was present, together with a population at smaller dimension, from few nanometers up to 311 approximately $50 \mathrm{~nm}$. After 7 days, the average size of the 312 bigger pores markedly decreased to values centered around 90313 $\mathrm{nm}$, whereas the smaller population remained unaltered in 314 terms of dimensions but increases in terms of volume, meaning 315 that the number of smaller pores increased. This is because of 316 the progressive formation of hydrated phases that produce the 317 increase of the surface area and of the total pore volume (see 318 Table 2)..$^{34}$ Later, at 15 days, similar pore size dimensions and 319 total surface area were found, with a small increase in the 320 number of bigger pores, which does not produce changes in 321 the value of the total pore volume (Table 2).

Considering the overall results obtained from the calori- 323 metric study, the wettability data, and the BET/BJH analysis, a 324 curing time of 7 days was considered appropriate for the 325 following investigations.

Quantification of Silicone Oil on the Surface. The 327 distribution of silicone oil film thickness on the infused cement 328 surfaces was quantified by AFM meniscus force measurements, 329 using a mapping technique recently developed by the 330 authors. ${ }^{16}$ The thickness of the lubricant film present on the 331 surface is directly related to the antifouling and slippery 332 performance of SLIPS. ${ }^{13}$ AFM force mapping experiments 333 reveal the local thickness of the lubricant with nanometric 334 resolution, at different stages after infusion, together with the 335 topography of the underlying substrate. When the tip 336 approaches the surface of the lubricant, there is an attractive 337 capillary effect, which continues as the tip moves through the 338 liquid. When the tip makes hard contact with the surface, a 339 strong repulsion is measured. The distance between the 340 attractive jump-in and the repulsive hard contact gives the 341 thickness of the lubricant while mapping the $z$-piezo position at 342 hard contact maps the topography. ${ }^{16}$ Figure 5 shows the $343 \mathrm{fs}$ 


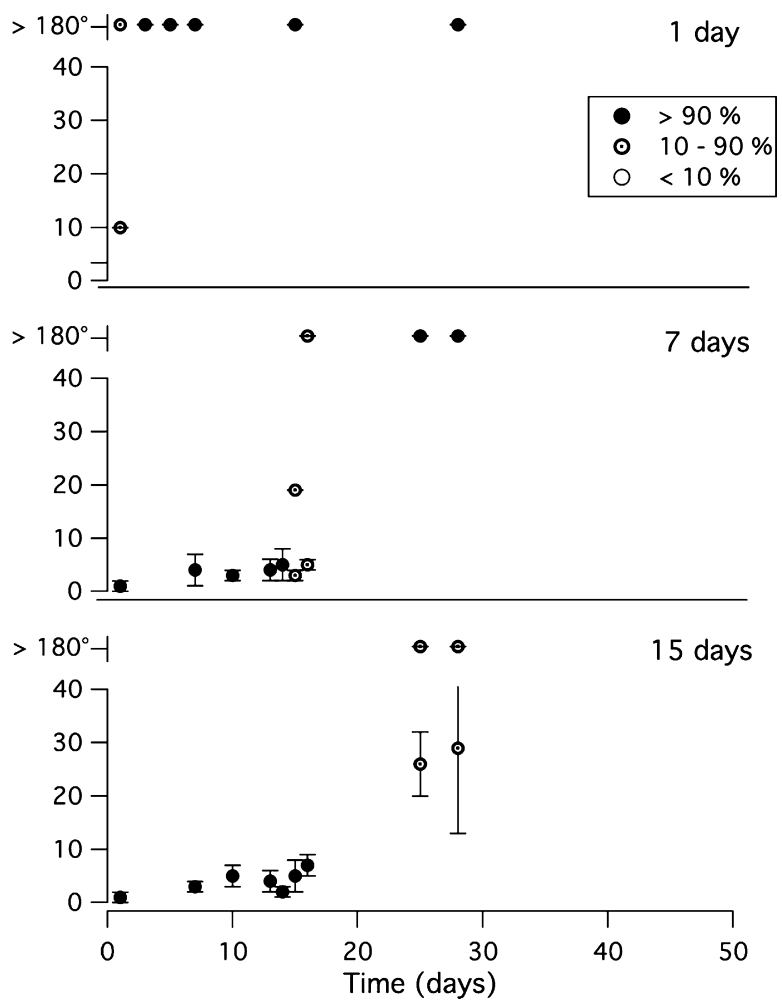

Figure 3. Sliding angle vs time since infusion for cement samples infused with 10k cSt silicone oil; the curing time of the cement prior to infusion is indicated in each panel. Full circles represent values measured with more than $90 \%$ incidence for that system; semifilled circles represent values measured with between 10 and $90 \%$ incidence; and empty circles represent less than $10 \%$ incidence related to the system.
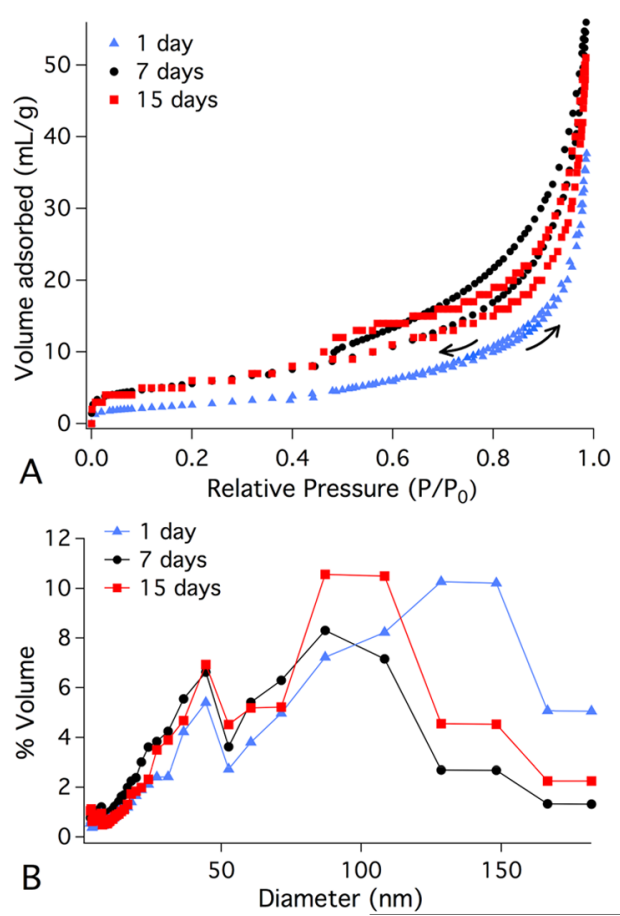

Figure 4. (A) Adsorption/desorption isotherms for cement samples cured for 1,7 , and 15 days; (B) distribution of pore volume percentage for cement samples at the same curing time, obtained by the $\mathrm{BJH}$ method.
Table 2. BET Surface Area and BJH Pore Volume Calculated from Data in Figure 4

\begin{tabular}{ccc} 
curing time (day) & BET surface area $\left(\mathrm{m}^{2} / \mathrm{g}\right)$ & total pore volume $(\mathrm{mL} / \mathrm{g})$ \\
1 & 9.4 & 0.05 \\
7 & 20.0 & 0.08 \\
15 & 22.2 & 0.07 \\
\hline
\end{tabular}

topography of a infused cement sample (part a1) and a map of 344 lubricant film thickness over the same area (part a2); the third 345 panel (a3) is the film thickness distribution over the whole 346 sample. The sample was cured for 1 week, silanised, and 347 infused with $10 \mathrm{k}$ cSt silicone oil, and then the meniscus force 348 measurements performed at 11,17 , and 40 days after lubricant 349 infusion. Immediately after infusion and for the first 10 days, 350 the thickness of the coating was more than $12 \mu \mathrm{m}$ (the 351 maximum measurable thickness, data not shown). From 11352 days onward, a thickness of about $1-2 \mu \mathrm{m}$ was found (Figure 353 $5 \mathrm{~A}$ ), with this situation corresponding to a highly slippery 354 surface, with a very low sliding angle (Figure 2). At 17 days 355 after infusion, the depletion became more pronounced, and 356 two populations of lubricant thickness appeared: there were 357 areas with a thickness of several hundred $\mathrm{nm}$ (Figure 5B) and 358 areas with lower thickness, mostly less than $200 \mathrm{~nm}$ (Figure 359 5C). Regions with different film thicknesses coexist, with high 360 features of the roughness becoming depleted of lubricants first. 361 These are the regions onto which the water droplets are likely 362 to remain pinned first, and, as shown in Figure 2, at this time, a 363 transition occurs from low to high values of sliding angle. 364 Depletion progressively increased over time because of the 365 absorption of the lubricant into the cement microstructure. 366 Finally, at 40 days after infusion (Figure 5D), a lubricant 367 thickness of less than $20 \mathrm{~nm}$ remained on the surface. Such a 368 low film thickness was not sufficient to maintain the slippery 369 properties, and indeed only high sliding angle values were 370 measured at this stage (Figure 2). The mechanism described 371 here is representative of the depletion of silicone oil occurring 372 with oils of different viscosities as well, although for higher 373 viscosity the timescale was slower (data not shown). 374

Water Permeability. The permeation of water in the 375 samples was monitored by low-temperature DSC, by 376 monitoring the depression of the freezing point of water 377 confined in the porous structure. ${ }^{33}$ When water is constrained 378 in pores with dimensions $\leq 1 \mathrm{~nm}$, it cannot be detected, but 379 when it is confined in larger pores, it can be quantified and 380 different populations can be distinguished: the smaller the 381 dimension of the pore, the lower the freezing temperature of 382 water confined into it. ${ }^{33}$ The comparison of samples whose 383 surfaces were treated or not gives information on the effect of 384 the surface treatment on the permeation of water into the 385 microstructure. Figure 6 shows the cooling thermograms for an $386 \mathrm{f} 6$ untreated cement sample, a sample untreated and immersed 387 into water for $24 \mathrm{~h}$, and a sample silanised, infused, and 388 immersed in water for $24 \mathrm{~h}$. The peaks observed as the samples 389 cooled in the range -10 to $-50{ }^{\circ} \mathrm{C}$ are due to the freezing of 390 the fraction of the water used to prepare the paste that has not 391 yet taken part in the hydration reaction and confined in pores 392 of different dimensions. ${ }^{32}$ The peak at about $-10^{\circ} \mathrm{C}$ is due to 393 the freezing of water confined in capillary pores, whose size is 394 large enough $(>12 \mathrm{~nm})$ to enable the water to freeze as "bulk", 395 unconfined water. The presence of water confined in the so- 396 called large gel pores, of size 3-12 nm, is revealed by the small 397 peak at about $-25{ }^{\circ} \mathrm{C}$. ${ }^{40,45}$ The peak at $-40{ }^{\circ} \mathrm{C}$ present in all 398 

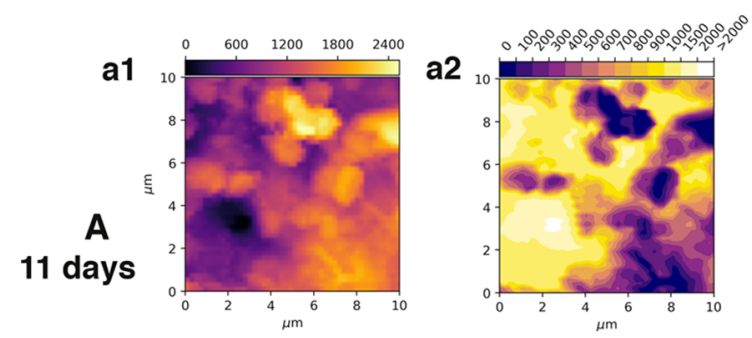

a3
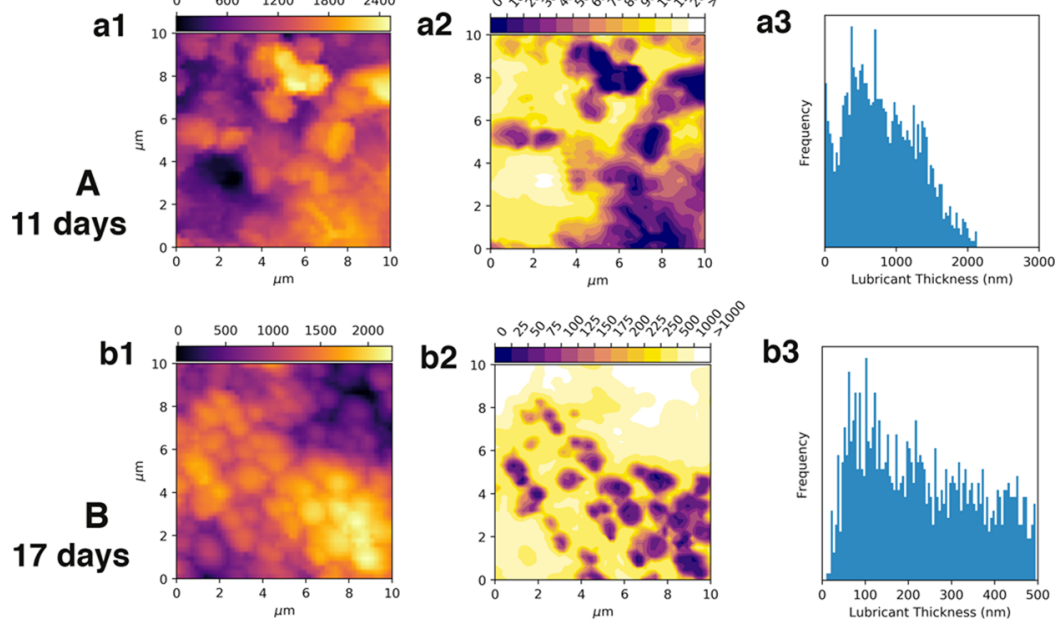

b3
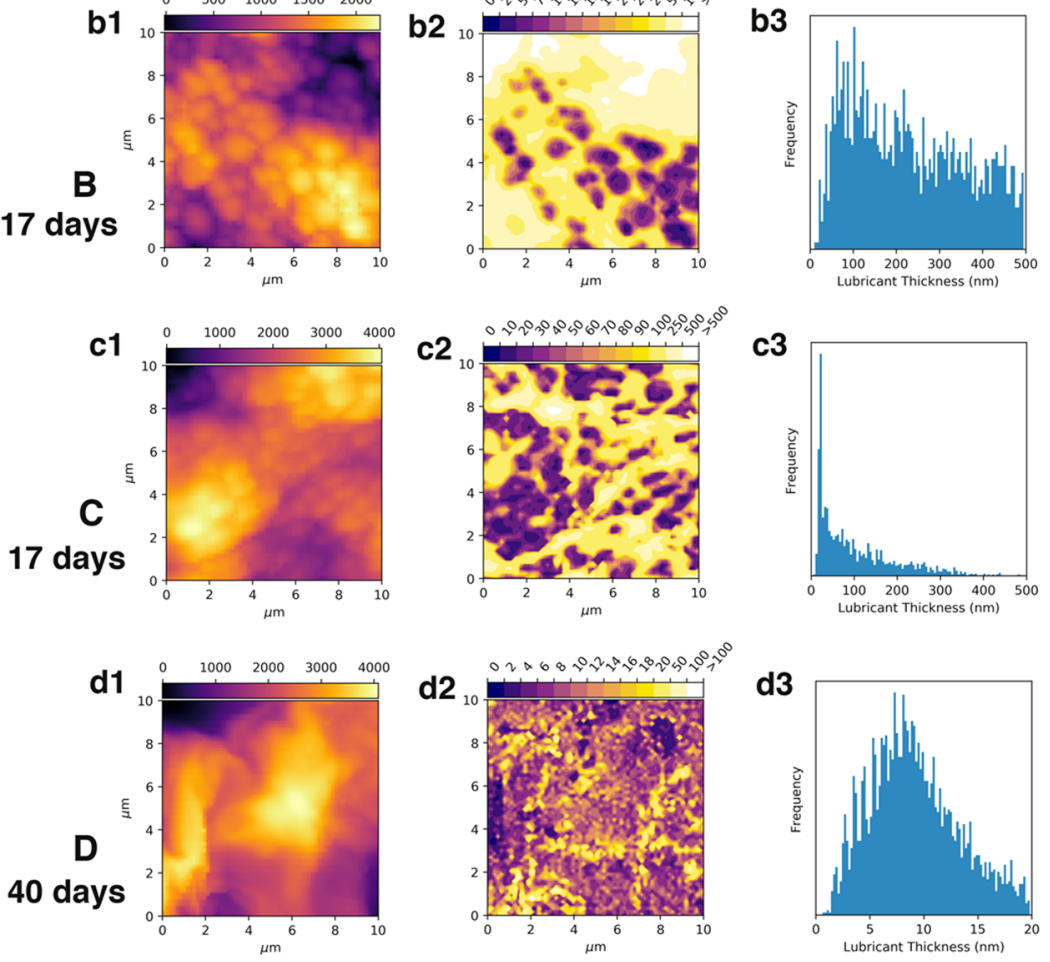

Figure 5. $(\mathrm{a} 1-\mathrm{d} 1)$ Topography of cement samples; $(\mathrm{a} 2-\mathrm{d} 2)$ map of lubricant film thickness over the same areas, measured by AFM meniscus force measurements; (a3-d3) distribution of lubricant film thickness. (A) 11, (B,C) 17, 17, and (D) 40 days.

399 the samples arises from the water confined in small gel pores $400(1-3 \mathrm{~nm})$. Each peak was integrated, and the areas were used 401 to calculate the amount of water involved. ${ }^{33}$ The signal at -54 $402{ }^{\circ} \mathrm{C}$ in the cooling scan of the treated sample is because of the 403 solidification of silicone oil, thus it was not considered.

404 Table 3 summarizes the amount of water permeated into the 405 different samples. Large differences were found concerning the 406 capillaries: no bulklike water was found in the reference sample 407 and, when immersed into water, the untreated and treated 408 samples differ. The infused surface had a lower amount of 409 water confined in capillary pores than the noninfused one (7.0 410 vs $11.8 \%$ ), which is a sign of a lower permeability. The 411 silanization and infusion treatment therefore reduced water 412 absorption in the infused cement. Nevertheless, as the test 413 consists of full immersion in water for $24 \mathrm{~h}$, only partial water 414 repellence was obtained.

415 Inhibition of Ice Nucleation. The freeze-thaw behavior 416 of a water droplet onto untreated and infused cement pastes 417 prepared into DSC pans was investigated to establish the effect 418 of infusion on ice nucleation. Following a protocol reported in 419 the literature, ${ }^{46}$ the samples were repeatedly cooled and 420 thawed. $^{47}$ The temperature at which the nucleation started was 421 measured from the freezing signals (Figure S4) and the 422 probability distribution was observed (Figure 7). The temper423 ature at which the samples froze $50 \%$ of the time (T50) represents the average nucleation temperature. Table S1 shows 424 a comparison of the nucleation temperature found for the 425 untreated and treated samples: the presence of lubricants 426 significantly increased the level of supercooling from around 427 $-9{ }^{\circ} \mathrm{C}$ for the untreated sample to $-27{ }^{\circ} \mathrm{C}$ for the infused 428 sample (an estimate of the error in the measurement is shown 429 in Table S1). This demonstrates that infusion of the samples 430 imparted icephobic properties onto cement.

431

\section{CONCLUSIONS}

432

The intrinsic roughness of cement paste was shown to be 433 suitable for effective trapping and infusion of silicone oil, 434 leading to high water repellency and slipperiness, effective for 435 several weeks, reduced water permeability, and icephobic 436 properties. The conclusions of this study are general and could 437 be applied to a number of porous materials where large pores 438 are present on the surface. Our main conclusions include: 439

1. The mechanism of the loss of lubricants in the cement- 440 like surface is mostly through penetration of the 441 lubricant in the bulk pore structure, through capillary 442 pores that reach the surface. Several approaches might 443 be suitable to reduce the loss of lubricants, including: 444 sealing the surface pores with a waxy material prior to 445 infusion; infusing the full volume of the porous material 446 


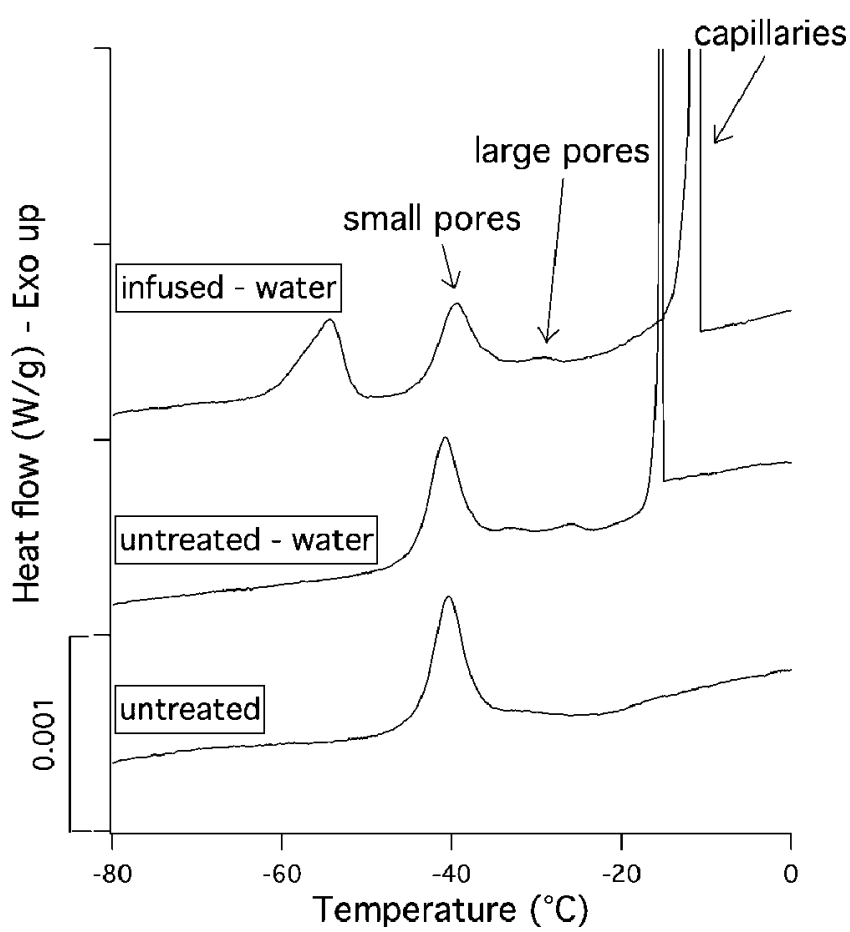

Figure 6. DSC thermograms of three cement pastes cured for 7 days: an untreated sample, a sample untreated and immersed in water for 24 $h$, and a sample infused with silicon oil and immersed in water for 24 h. The cooling scans are offset for clarity.

Table 3. Amount of Water Freezing at Different Temperature Ranges

\begin{tabular}{|c|c|c|c|}
\hline \multirow[b]{2}{*}{ sample } & \multicolumn{3}{|c|}{ water amount $(\%)^{a}$} \\
\hline & $\begin{array}{l}\text { capillary } \\
\text { pores }\end{array}$ & $\begin{array}{l}\text { large pores } \\
(3-12 \mathrm{~nm})^{c}\end{array}$ & $\begin{array}{l}\text { small pores } \\
(1-3 \mathrm{~nm})^{d}\end{array}$ \\
\hline untreated & 0 & $0.2 \pm 0.1$ & $1.9 \pm 0.5$ \\
\hline untreated-water & $11.8 \pm 2.9$ & $0.3 \pm 0.1$ & $1.8 \pm 0.5$ \\
\hline infused-water & $7.0 \pm 1.8$ & $0.3 \pm 0.1$ & $1.1 \pm 0.3$ \\
\hline
\end{tabular}

${ }^{a}$ The percentages are calculated with respect to the total weight of the paste. ${ }^{b}$ Region of bulk-like water confined in capillaries: water freezing from -5 to $-20{ }^{\circ} \mathrm{C}$. ${ }^{c}$ Water freezing between -20 and $-35{ }^{\circ} \mathrm{C}$. ${ }^{d}$ Water freezing between -35 and $-50{ }^{\circ} \mathrm{C}$. with the lubricant (as long as the thickness of the cement layer is small); and replenishing the lubricant at regular (4 weeks) intervals.

2. The cement curing time affects the success of infusion, as the pore size is highly dependent on curing time. Cement surfaces needed to be cured for a minimum of 7 days, until the microstructure had mostly formed, before infusion could be successfully conducted. At this point, the highest distribution of pores was around $90 \mathrm{~nm}$ with a smaller population of around $40 \mathrm{~nm}$. The capillary pores were the ones that most benefited from infusion in reducing water penetration when samples were fully immersed in water.

3. The viscosity of the lubricant affects the efficiency of infusion. While in general a lower viscosity lubricant naturally leads to more slippery surfaces and faster rates of roll-off of droplets, in highly porous surfaces such as these, higher viscosity lubricants are required, as they sufficiently delay lubricant depletion via penetration into the material to make the surfaces practically useful.
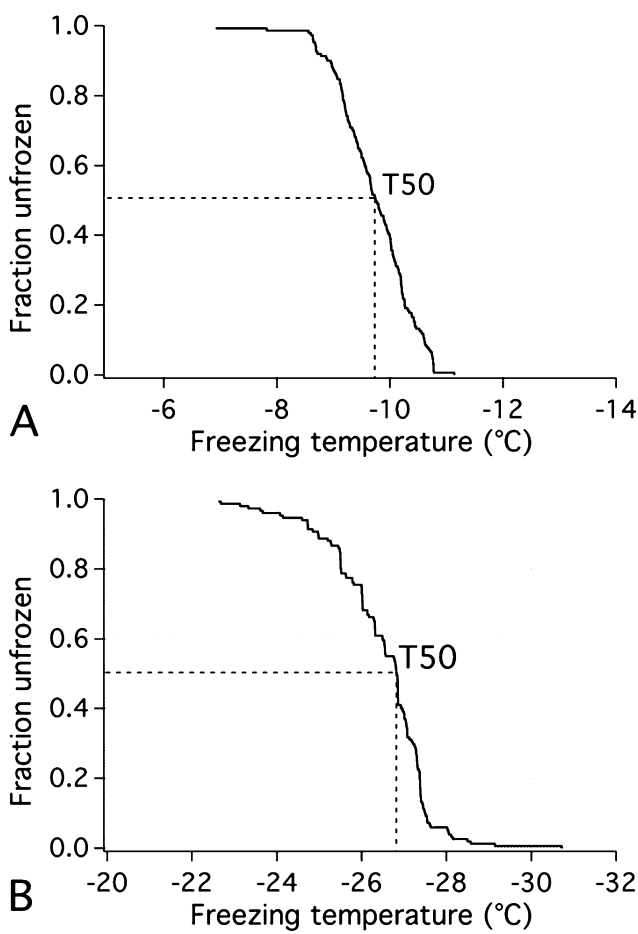

Figure 7. Probability distribution curves for the event of freezing for the untreated cement (A) and infused cement (B).

While low viscosity lubricants $(<10 \mathrm{k} c \mathrm{ct})$ resulted in 467 poor retention of the lubricant on the cement surface, 468 because of the quick absorption in the cement porosity, 469 higher viscosity lubricants ( $10 \mathrm{k}$ and $20 \mathrm{k} \mathrm{cSt}$ ) resulted in 470 good slippery properties, maintained for over 4 weeks. 471

4. Even in surfaces with high, random, and self-assembled 472 roughness such as those studied here, the slippery 473 properties can be easily related to the amount of 474 lubricants present at each location of the surface, rather 475 than to the surface structure itself. Slippery properties 476 were observed on cement surfaces when lubricant 477 thickness was of the order of hundreds of nm. Below 478 this value of lubricant thickness, water droplets started to 479 pin on the micro- and nanoscale roughness of the 480 surface. AFM meniscus force mapping pioneered by us is 481 invaluable in relating how lubricant thickness and 482 redistribution over time affect the surface performance. 483

5. To the best of our knowledge, the permeation of water 484 into SLIPS-treated surfaces was quantified for the first 485 time by low-temperature DSC. DSC also allowed, for the 486 first time, evaluating the icephobic properties of surfaces. 487 Cement surfaces represented a case study and this 488 method is likely to be generalizable to the study of many 489 other pour material and ice nucleation on surfaces. 490 Lubricant-infused surfaces showed lower permeability 491 than noninfused ones, with the lubricant reducing water 492 absorption into capillaries. Finally, slippery cement- 493 based materials significantly lowered the freezing 494 temperature of water down to $-27^{\circ} \mathrm{C}$.

495

Given the random nature of cement roughness and the 496 presence of large pores on the surface, it is remarkable that 497 infusion was successful to this reported extent. Cement is an 498 industrially relevant material, with broad areas of interest and a 499 significant economic impact. Although further improvements 500 are necessary for the obtainment of a durable solution, this 501 
502 paper could contribute to develop a wide spectrum of fields, 503 from reducing the impact of graffiti to reducing the damage by 504 marine spray and by acid rain. This work extends the potential 505 use of lubricant infusion to real-world materials and to surfaces 506 with nonideal roughness.

\section{ASSOCIATED CONTENT}

\section{S Supporting Information}

509 The Supporting Information is available free of charge on the 510 ACS Publications website at DOI: 10.1021/acs.jpcc.8b11221.

511 Water static contact angle versus time since infusion, 512 using silicone oils of different viscosity, free water index 513 as a function of the curing time, water static contact 514 angle versus time since infusion for infused cement 515 samples, freezing temperature of a water droplet onto 516 untreated and silanised and infused samples, and 517 comparison of water supercooling for surfaces untreated 518 and treated (PDF)

\section{AUTHOR INFORMATION}

\section{Corresponding Author}

521 *E-mail: chiara.neto@sydney.edu.au. Phone: +61 293512752. 522 ORCID $\odot$

523 Chiara Neto: 0000-0001-6058-0885

\section{Present Address}

${ }_{525}{ }^{\S}$ Department of Chemistry "Ugo Schiff” \& CSGI, University of 526 Florence, Sesto Fiorentino 50019, Florence, Italy.

\section{Author Contributions}

528 The manuscript was written through contributions of all 529 authors. All authors have given approval to the final version of 530 the manuscript.

531 Notes

532 The authors declare no competing financial interest.

\section{$533 \square$ ACKNOWLEDGMENTS}

534 The authors thank the Australian Research Council, The 535 University of Sydney, the Consorzio Interuniversitario per lo 536 Sviluppo dei Sistemi a Grande Interfase (CSGI) and the 537 Erasmus Mundus NANOPHI Project for funding. Prof. 538 Gianluca Ranzi and Osvaldo Vallati are warmly thanked for 539 providing of the cement powders and for useful discussions.

\section{$540 \square$ ABBREVIATIONS}

541 SLIPS, slippery liquid infused surfaces; DSC, differential 542 scanning calorimetry; BJH, Barrett-Joyner-Halenda method

\section{$543 \square$ REFERENCES}

544 (1) Scarratt, L. R. J.; Steiner, U.; Neto, C. A Review on the 545 Mechanical and Thermodynamic Robustness of Superhydrophobic 546 Surfaces. Adv. Colloid Interface Sci. 2017, 246, 133-152.

547 (2) Owais, A.; Smith-Palmer, T.; Gentle, A.; Neto, C. Influence of 548 Long-Range Forces and Capillarity on the Function of Underwater 549 Superoleophobic Wrinkled Surfaces. Soft Matter 2018, 14, 66275506634

551 (3) Wong, T.-S.; Kang, S. H.; Tang, S. K. Y.; Smythe, E. J.; Hatton, 552 B. D.; Grinthal, A.; Aizenberg, J. Bioinspired Self-Repairing Slippery 553 Surfaces with Pressure-Stable Omniphobicity. Nature 2011, 477, $554443-447$.

555 (4) Zhang, J.; Gu, C.; Tu, J. Robust Slippery Coating with Superior 556 Corrosion Resistance and Anti-Icing Performance for AZ31B Mg 557 Alloy Protection. ACS Appl. Mater. Interfaces 2017, 9, 11247-11257.
(5) Yeong, Y. H.; Wang, C.; Wynne, K. J.; Gupta, M. C. Oil-Infused 558 Superhydrophobic Silicone Material for Low Ice Adhesion with Long- 559 Term Infusion Stability. ACS Appl. Mater. Interfaces 2016, 8, 32050- 560 32059.

(6) Kim, P.; Wong, T.-S.; Alvarenga, J.; Kreder, M. J.; Adorno- 562 Martinez, W. E.; Aizenberg, J. Liquid-Infused Nanostructured 563 Surfaces with Extreme Anti-Ice and Anti-Frost Performance. ACS 564 Nano 2012, 6, 6569-6577.

(7) Liu, Q.; Yang, Y.; Huang, M.; Zhou, Y.; Liu, Y.; Liang, X. 566 Durability of a Lubricant-Infused Electrospray Silicon Rubber Surface 567 as an Anti-Icing Coating. Appl. Surf. Sci. 2015, 346, 68-76. 568

(8) Subramanyam, S. B.; Rykaczewski, K.; Varanasi, K. K. Ice 569 Adhesion on Lubricant-Impregnated Textured Surfaces. Langmuir 570 2013, 29, 13414-13418.

(9) Yin, X.; Zhang, Y.; Wang, D.; Liu, Z.; Liu, Y.; Pei, X.; Yu, B.; 572 Zhou, F. Integration of Self-Lubrication and Near-Infrared Photo- 573 thermogenesis for Excellent Anti-Icing/Deicing Performance. Adv. 574 Funct. Mater. 2015, 25, 4237-4245. 575

(10) Zhu, L.; Xue, J.; Wang, Y.; Chen, Q.; Ding, J.; Wang, Q. Ice- 576 Phobic Coatings Based on Silicon-Oil-Infused Polydimethylsiloxane. 577 ACS Appl. Mater. Interfaces 2013, 5, 4053-4062.

(11) Wang, P.; Zhang, D.; Lu, Z.; Sun, S. Fabrication of Slippery 579 Lubricant-Infused Porous Surface for Inhibition of Microbially 580 Influenced Corrosion. ACS Appl. Mater. Interfaces 2016, 8, 1120- 581 1127.

(12) MacCallum, N.; Howell, C.; Kim, P.; Sun, D.; Friedlander, R.; 583 Ranisau, J.; Ahanotu, O.; Lin, J. J.; Vena, A.; Hatton, B.; et al. Liquid- 584 Infused Silicone As a Biofouling-Free Medical Material. ACS Biomater. 585 Sci. Eng. 2014, 1, 43-51.

(13) Ware, C. S.; Smith-Palmer, T.; Peppou-Chapman, S.; Scarratt, 587 L. R. J.; Humphries, E. M.; Balzer, D.; Neto, C. Marine Antifouling 588 Behavior of Lubricant-Infused Nanowrinkled Polymeric Surfaces. ACS 589 Appl. Mater. Interfaces 2018, 10, 4173-4182.

(14) Wang, P.; Zhang, D.; Sun, S.; Li, T.; Sun, Y. Fabrication of 591 Slippery Lubricant-Infused Porous Surface with High Underwater 592 Transparency for the Control of Marine Biofouling. ACS Appl. Mater. 593 Interfaces 2016, 9, 972-982. 594

(15) Wei, C.; Zhang, G.; Zhang, Q.; Zhan, X.; Chen, F. Silicone Oil- 595 Infused Slippery Surfaces Based on Sol-Gel Process-Induced Nano- 596 composite Coatings: A Facile Approach to Highly Stable Bioinspired 597 Surface for Biofouling Resistance. ACS Appl. Mater. Interfaces 2016, 8, 598 34810-34819.

(16) Peppou-Chapman, S.; Neto, C. Mapping Depletion of 600 Lubricant Films on Antibiofouling Wrinkled Slippery Surfaces. ACS 601 Appl. Mater. Interfaces 2018, 10, 33669-33677. 602

(17) Smith, J. D.; Dhiman, R.; Anand, S.; Reza-Garduno, E.; Cohen, 603 R. E.; McKinley, G. H.; Varanasi, K. K. Droplet Mobility on 604 Lubricant-Impregnated Surfaces. Soft Matter 2013, 9, 1772-1780. 605

(18) Preston, D. J.; Song, Y.; Lu, Z.; Antao, D. S.; Wang, E. N. 606 Design of Lubricant Infused Surfaces. ACS Appl. Mater. Interfaces 607 2017, 9, 42383-42392.

(19) Kim, P.; Kreder, M. J.; Alvarenga, J.; Aizenberg, J. Hierarchical 609 or Not? Effect of the Length Scale and Hierarchy of the Surface 610 Roughness on Omniphobicity of Lubricant-Infused Substrates. Nano 611 Lett. 2013, 13, 1793-1799.

612

(20) Leslie, D. C.; Waterhouse, A.; Berthet, J. B.; Valentin, T. M.; 613 Watters, A. L.; Jain, A.; Kim, P.; Hatton, B. D.; Nedder, A.; Donovan, 614 K.; et al. A Bioinspired Omniphobic Surface Coating on Medical 615 Devices Prevents Thrombosis and Biofouling. Nat. Biotechnol. 2014, 616 32, 1134-1140.

(21) Yuan, S.; Li, Z.; Song, L.; Shi, H.; Luan, S.; Yin, J. Liquid- 618 Infused Poly(styrene-b-isobutylene-b-styrene) Microfiber Coating 619 Prevents Bacterial Attachment and Thrombosis. ACS Appl. Mater. 620 Interfaces 2016, 8, 21214-21220.

(22) Manabe, K.; Kyung, K.-H.; Shiratori, S. Biocompatible Slippery 622 Fluid-Infused Films Composed of Chitosan and Alginate via Layer-by- 623 Layer Self-Assembly and Their Antithrombogenicity. ACS Appl. 624 Mater. Interfaces 2015, 7, 4763-4771. 
626 (23) Damasceni, A.; Dei, L.; Fratini, E.; Ridi, F.; Chen, S.-H.; 627 Baglioni, P. A Novel Approach Based on Differential Scanning 628 Calorimetry Applied to the Study of Tricalcium Silicate Hydration 629 Kinetics †. J. Phys. Chem. B 2002, 106, 11572-11578.

630 (24) Ridi, F.; Dei, L.; Fratini, E.; Chen, S.-H.; Baglioni, P. Hydration 631 Kinetics of Tri-Calcium Silicate in the Presence of Superplasticizers. J. 632 Phys. Chem. B 2003, 107, 1056-1061.

633 (25) Muhammad, N. Z.; Keyvanfar, A.; Majid, M. Z. A.; Shafaghat, 634 A.; Mirza, J. Waterproof Performance of Concrete: A Critical Review 635 on Implemented Approaches. Constr. Build. Mater. 2015, 101, 80-90. 636 (26) Han, B.; Zhang, L.; Ou, J. Hydrophobic/Superhydrophobic 637 Concrete. Smart and Multifunctional Concrete Toward Sustainable 638 Infrastructures; Springer Singapore: Singapore, 2017; pp 339-357. 639 (27) Wittmann, F. H.; Wittmann, A. D. A.; Wang, P. G. Capillary 640 Absorption of Integral Water Repellent and Surface Impregnated 641 Concrete. Restor. Build. Monum. 2014, 20, 281-290.

642 (28) de Vries, I. J.; Polder, R. B. Hydrophobic Treatment of 643 Concrete. Constr. Build. Mater. 1997, 11, 259-265.

644 (29) Ridi, F.; Fratini, E.; Luciani, P.; Winnefeld, F.; Baglioni, P. 645 Hydration Kinetics of Tricalcium Silicate by Calorimetric Methods. J. 646 Colloid Interface Sci. 2011, 364, 118-124.

647 (30) Sagiv, J. Organized Monolayers by Adsorption. 1. Formation 648 and Structure of Oleophobic Mixed Monolayers on Solid Surfaces. J. 649 Am. Chem. Soc. 1980, 102, 92-98.

650 (31) Gotz, J.; Weisser, H. Correlation of the Viscosity and the 651 Molecular Weight of Silicone Oils with the $\mathrm{T}_{2}$ NMR Relaxation 652 Times. Organosilicon Chemistry V; Wiley, 2008; pp 584-594.

653 (32) Ridi, F.; Fratini, E.; Luciani, P.; Winnefeld, F.; Baglioni, P. 654 Hydration Kinetics of Tricalcium Silicate by Calorimetric Methods. J. 655 Colloid Interface Sci. 2011, 364, 118-124.

656 (33) Ridi, F.; Luciani, P.; Fratini, E.; Baglioni, P. Water Confined in 657 Cement Pastes as a Probe of Cement Microstructure Evolution. J. 658 Phys. Chem. B 2009, 113, 3080-3087.

659 (34) Ridi, F.; Fratini, E.; Baglioni, P. Fractal Structure Evolution 660 during Cement Hydration by Differential Scanning Calorimetry: 661 Effect of Organic Additives. J. Phys. Chem. C 2013, 117, 2547866225487.

663 (35) Hansen, E. W.; Gran, H. C.; Sellevold, E. J. Heat of Fusion and 664 Surface Tension of Solids Confined in Porous Materials Derived from 665 a Combined Use of NMR and Calorimetry. J. Phys. Chem. B 1997, $666101,7027-7032$.

667 (36) Wilson, P. W.; Heneghan, A. F.; Haymet, A. D. J. Ice 668 Nucleation in Nature: Supercooling Point (SCP) Measurements and 669 the Role of Heterogeneous Nucleation. Cryobiology 2003, 46, 88-98. 670 (37) Heneghan, A. F.; Wilson, P. W.; Haymet, A. D. J. 671 Heterogeneous Nucleation of Supercooled Water, and the Effect of 672 an Added Catalyst. Proc. Natl. Acad. Sci. U.S.A. 2002, 99, 9631-9634. 673 (38) Stewart, A.; Schlosser, B.; Douglas, E. P. Surface Modification 674 of Cured Cement Pastes by Silane Coupling Agents. ACS Appl. Mater. 675 Interfaces 2013, 5, 1218-1225.

676 (39) Daniel, D.; Timonen, J. V. I.; Li, R.; Velling, S. J.; Aizenberg, J. 677 Oleoplaning Droplets on Lubricated Surfaces. Nat. Phys. 2017, 13, 678 1020-1025.

679 (40) Jennings, H. M. Refinements to Colloid Model of C-S-H in 680 Cement: CM-II. Cem. Concr. Res. 2008, 38, 275-289.

681 (41) Pane, I.; Hansen, W. Investigation of Blended Cement 682 Hydration by Isothermal Calorimetry and Thermal Analysis. Cem. 683 Concr. Res. 2005, 35, 1155-1164.

684 (42) Giles, C. H.; Smith, D.; Huitson, A. A general treatment and 685 classification of the solute adsorption isotherm. I. Theoretical. J. 686 Colloid Interface Sci. 1974, 47, 755-765.

687 (43) Sing, K. S. W.; Everett, D. H.; Haul, R. A. W.; Moscou, L.; 688 Pierotti, R. A.; Rouquerol, J.; Siemieniewska, T. Reporting 689 Physisorption Data for Gas/Solid Systems. Pure Appl. Chem. 1985, $69057,603-619$.

691 (44) Thommes, M.; Kaneko, K.; Neimark, A. V.; Olivier, J. P.; 692 Rodriguez-Reinoso, F.; Rouquerol, J.; Sing, K. S. W. Physisorption of 693 Gases, with Special Reference to the Evaluation of Surface Area and
Pore Size Distribution (IUPAC Technical Report). Pure Appl. Chem. 694 2015, 87, 1051-1069.

(45) Jennings, H. M.; Bullard, J. W.; Thomas, J. J.; Andrade, J. E.; 696 Chen, J. J.; Scherer, G. W. Characterization and Modeling of Pores 697 and Surfaces in Cement Paste. J. Adv. Concr. Technol. 2008, 6, 5-29. 698

(46) Wilson, P. W.; Lu, W.; Xu, H.; Kim, P.; Kreder, M. J.; 699 Alvarenga, J.; Aizenberg, J. Inhibition of Ice Nucleation by Slippery 700 Liquid-Infused Porous Surfaces (SLIPS). Phys. Chem. Chem. Phys. 701 2013, 15, 581-585.

(47) Monteilhet, L.; Korb, J.-P.; Mitchell, J.; McDonald, P. J. 703 Observation of Exchange of Micropore Water in Cement Pastes by 704 Two-Dimensional T 2 - T 2 Nuclear Magnetic Resonance 705 Relaxometry. Phys. Rev. E: Stat., Nonlinear, Soft Matter Phys. 2006, 706 74, 061404. 